\title{
Theoretical Studies of Flavor-Changing Neutral Currents in 1980's
}

\author{
C.S. Lim* \\ Department of Physics, Kobe University, Kobe 657-8501, Japan \\ E-mail: limekobe-u.ac.jp
}

\begin{abstract}
Flavor physics in 1980's is briefly summarized. It is pointed out that the question of whether heavy fermions decouple from low energy processes or not led to formulae which are valid for arbitrarily heavy intermediate fermions. Such heavy particles were shown to have non-decoupling contributions to flavor-changing neutral current (FCNC) processes. In particular, the top quark turned out to be unexpectedly heavy, and was found to have important non-decoupling contributions to FCNC processes, including CP violating processes, as the reflection of the spontaneous gauge symmetry breaking. Recent claims on the possible deviations of the rates of some FCNC processes from the Standard Model predictions may signal some "new physics". It is argued that if we take the possibility of the 4th generation as a candidate of the new physics, the unknown heavy fermions of 4th generation are expected to have enhancement factor proportional to $m_{t^{\prime}}^{2}$ in their non-decoupling contributions to the processes, and may be responsible for such deviations.
\end{abstract}

2009 KAON International Conference KAON09,

June 09 - 122009

Tsukuba, Japan

\footnotetext{
* Speaker.
} 


\section{A little (biased) history of flavor physics mainly in 1980's}

I would like to mention very briefly a little biased history of flavor physics in 1980's, viewed mainly through the work in collaboration with Prof. Takeo Inami on the effects of heavy fermions in the Flavor-Changing Neutral Current (FCNC) processes, "Effects of Superheavy Quarks and Leptons in Low-Energy Weak Processes $K_{L} \rightarrow \mu \bar{\mu}, K^{+} \rightarrow \pi^{+} v \bar{v}$ and $K^{0} \leftrightarrow \bar{K}^{0 "}$ 《1].

Suppose there is a perfect symmetry among generations (or flavors), then the generation number or flavor is exactly conserved, as Noether's theorem suggests and there will be no FlavorChanging Neutral Current (FCNC) processes. Thus FCNC processes stem only from the violation of flavor symmetry due to the mass differences of fermions belonging to different generations together with flavor mixings.

The Weinberg-Salam model was originally formulated with only 3 flavors, $u, d, s$, thus explicitly breaking the generation symmetry, which led to the problem of too rapid FCNC processes. It is well-known that to remedy the problem Glashow, Iliopoulos and Maiani introduced the c quark, thus realizing the symmetry between 1 st and 2 nd generations, though the symmetry is not perfect because of the quark mass differences (GIM mechanism) [2].

FCNC processes are sensitive to the breaking of the flavor symmetry due to the mass-squared differences among quarks, which still remained even after the introduction of $\mathrm{c}$ quark. From such view point, M.K. Gaillard and B.W. Lee could predict, in their pioneering work [3], the charm quark mass $m_{c}$ to be $\simeq 1.5(\mathrm{GeV})$ before the discovery of $J / \Psi$ by comparing the theoretical predictions on the FCNC processes as the functions of $m_{c}\left(\theta_{C}\right.$ was known) with the data. Their work is an ideal one in the field of particle phenomenology, I believe, and greatly inspired our work on the FCNC processes.

Then, what about the third up-type quark, top quark, introduced by Kobayashi and Maskawa in their renowned paper which was awarded the Nobel prize of 2008 [4] ? In early 80's, t quark was somehow regarded to be not so heavy, say $m_{t} \leq 30(\mathrm{GeV})$. Then, the formulae by GaillardLee, which utilized the approximation $m_{q} \ll M_{W}$ ( $m_{q}$ : up-type quark masses), still seemed to give reasonable results for FCNC processes.

However, Takeo Inami and myself published a paper [1], in which, in contrast to the common sense at that time, we assumed the presence of "arbitrarily" heavy (but not so heavy as to spoil the perturbative expansion by too large Yukawa couplings) quarks and leptons appearing as the virtual states in the FCNC processes. Though the heavy fermions can be any fermions of sequential type, what we had in our mind were, e.g., $\mathrm{t}$ quark, the up-type quark $t^{\prime}$ or heavy lepton $L$ of possible (?) 4th generation. We derived several functions describing the quantum effects of these heavy fermions to the representative FCNC processes, without invoking the approximation $m_{q} \ll M_{W}$, which is no longer valid.

\subsection{A strong theoretical motivation}

The reason why we assumed the presence of such "super-heavy" fermions, whose masses $m_{f} \gg M_{W}$, was that we had a strong theoretical motivation . Namely, we were eager to know whether such heavy fermions provide some non-decoupling effects, as is suggested by the large Yukawa couplings of heavy fermions. Let us note that in the theories with spontaneous symmetry 
breaking (SSB), such as the standard model, large masses provided by the SSB mean large couplings with the Higgs. Such large couplings (but not so large as to spoil the perturbative expansion) will contribute to the numerators of the transition amplitudes, thus making the non-decoupling phenomena plausible. Once such non-decoupling effects are confirmed to exist, they will yield very useful information of the properties of the heavy particles before their direct production in high energy accelerators.

Luckily enough for us, the $t$ quark turned out to be unexpectedly heavy, i.e. $m_{t} \simeq 175(\mathrm{GeV})>$ $M_{W}$. Hence, our formulae became relevant to describe the FCNC and also closely related CP violating processes.

We worked in the $R_{\xi}$ gauge [5]. In this gauge, each diagram has $\xi$-dependence which makes the computations rather cumbersome. The fact that the final result should be gauge invariant and therefore $\xi$-independent helps to check the correctness of the calculations. In this gauge, the (would-be) Nambu-Goldstone bosons have Yukawa couplings, which are proportional to the masses of intermediate heavy fermions, leading to the possibility of non-decoupling effects of heavy fermions.

\subsection{A useful suggestion by Prof. K. Fujikawa}

In the process of the calculation, we found that the $\xi$-dependence appearing in the FCNC Zvertex gave $\xi$-dependent 4-fermi effective lagrangian, which are relevant for the decay processes $K_{L} \rightarrow \mu \bar{\mu}, K^{+} \rightarrow \pi^{+} v \bar{v}$ (see Fig (1). The piece with $V-A$ type couplings of leptons has no problem, since the $\xi$-dependence was known to be exactly cancelled by the corresponding $\xi$-dependence in the box diagram which was in our mind as the diagram to be evaluated. The problem was the piece with vector-like coupling of charged lepton $\mu$, which seemed to have no counterpart to cancel the $\xi$-dependence.

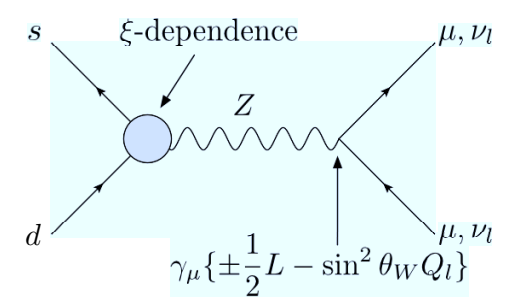

Figure 1: The contribution of FCNC Z-vertex with $\xi$-dependence

Soon we realized that the problem would probably solved by adding "QED-penguin" diagram (Fig,2), though the diagram itself has no contributions to the decay processes. But, I was a little reluctant to start the calculation, since to get the QED-penguin diagram it was necessary to include the external momentum, which could be ignored in the evaluation of Z-penguin or box diagrams. Then, I asked Prof. Kazuo Fujikawa his advise as the expert in the field and he recommended me to calculate the $\bar{s} d \gamma$ vertex as well for the completeness, even though it does not contribute to the relevant FCNC processes.

So we decided to calculate the $\bar{s} d \gamma$ vertex and the obtained vertex functions ( $F_{1}$ and $F_{2}$ in our notation) were later used in the analysis of FCNC processes, such as $b \rightarrow s \gamma$ or $b \rightarrow s l^{+} l^{-}$. We 


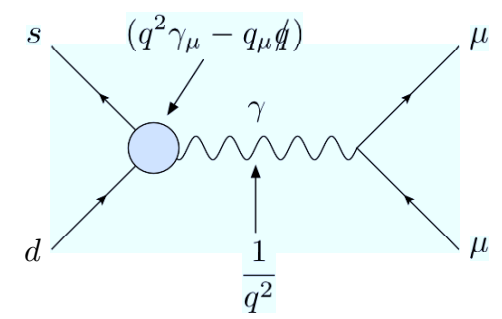

Figure 2: The "QED-penguin" diagram

greatly appreciate the useful advice by Prof. Fujikawa. By the way, the QED-penguin diagram has $\frac{1}{q^{2}}$ due to the photon propagator and seems to be impossible to cancel the $\xi$-dependence in the Zexchange diagram, which does not have such a pole. What happens is that such pole is cancelled by the factor $q^{2}$ appearing in the FCNC photon vertex, as is explained in Fig 2 and the contributions of the QED-penguin and Z-exchange diagrams become comparable.

\subsection{Attempts to estimate $m_{t}$}

Once exact formulae to describe FCNC processes are obtained, it is interesting to ask whether the estimation of $m_{t}$ is possible by comparing the formulae with the corresponding data. In fact, A.J. Buras attempted to estimate the upper bound on $m_{t}$ [6]. Because of large uncertainties of mixing angles and $\mathrm{CP}$ phase at that time the estimation was a challenging issue. We would like to thank Prof. Buras for bringing our work to public attention.

\section{Non-decoupling effects due to heavy fermions}

When new theories are proposed, they usually predict the presence of some characteristic unknown heavy particles, such as super-partners in supersymmetric theories. It is then of crucial importance whether such heavy particles manifest themselves in low energy processes. There exists a "decoupling theorem" by T. Appelquist and J. Carazzone [7], saying that the effects of heavy particles are suppressed by the inverse powers of their large masses. The theorem was proved in QED-like theories and is known to be valid for theories without spontaneous breaking of gauge symmetries.

At the tree level, the theorem is easily understood to hold, since heavy particles appear only in the intermediate states of Feynman diagrams and their propagators are simply suppressed by the inverse powers of their masses. For instance, the well-known "see-saw mechanism" proposed by Gell-Mann, Ramond, Slansky and Yanagida,

$$
m_{L} \simeq \frac{m_{D}^{2}}{m_{R}} \quad\left(m_{L}, m_{R}: \text { left- and right-handed Majorana masses, } m_{D}: \text { Dirac mass }\right),
$$

may be understood as the implication of the decoupling of heavy right-handed neutrinos. The Majorana mass term for the left-handed neutrino does not exist as a renormalizable operator in the theory having only Higgs doublet, such as the standard model, and is described in terms of a gauge 
invariant operator with higher $(>4)$ mass dimension, which is obtained from the Feynman diagram shown in Fig.3,

$$
\propto \frac{1}{m_{R}}\left(\phi^{\dagger} L\right)^{2},
$$

where $\phi$ and $L$ denote Higgs and lepton doublets. The Majorana mass of right-handed neutrino $m_{R}$, being invariant under the gauge transformation of the standard model, reflects the scale of some new physics (NP). $m_{R} \gg M_{W}$ then leads to the decoupling of right-handed neutrino and left-handed neutrino loses the chiral-partner to form a Dirac mass.

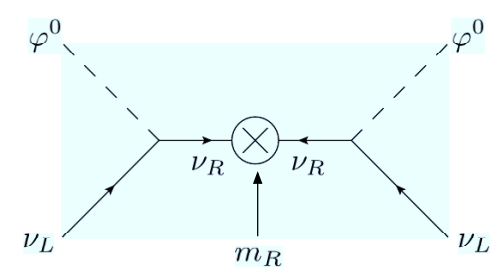

Figure 3: Feynman diagram contributing to the left-handed Majorana masses for neutrinos

At the quantum level, however, the decoupling is not trivial, as the energy of intermediate states can be arbitrarily large because of uncertainty relation. In fact, in QED assuming the presence of a heavy lepton with mass $\mathrm{M}$, it does contribute to the renormalization factor, as

$$
\log \left(\frac{\Lambda}{M}\right) \quad(\Lambda: \text { cutoff })
$$

After the renormalization procedure, however, all remaining observables (described by operators with higher mass dimensions) are known to be suppressed by the powers of $\frac{1}{M}$ [7].

In gauge theories with spontaneous symmetry breaking (SSB), the situation can be quite different. When the large masses of heavy particles are provided not by some gauge singlet NP mass scale $M$ but from the VEV of the Higgs field, i.e. from the weak scale $M_{W}$ itself, the large masses mean "strong" coupling with the Higgs particle. Since such "large" couplings appear in the numerator of amplitude, there may be no suppression by the powers of $\frac{1}{M}$. Thus, it will be reasonable to expect some non-decoupling effects.

In fact, in our work [1], we found non-decoupling effects of heavy fermions. For instance, the contributions of $t$ quark in FCNC processes turned out to grow up as

$$
\propto m_{t}^{2}, \quad \log \left(\frac{m_{t}}{m_{u, c}}\right)
$$

The reason to get such non-decoupling effects can be explained as follows. As an example, take the box diagram shown in Fig.4. The exchange of $\mathrm{N}-\mathrm{G}$ boson $\varphi^{ \pm}$and $\mathrm{t}$ quark is expected to yield a contribution, which is proportional to the 4th order of the top-Yukawa coupling (assuming $m_{t} \gg M_{W}$ for simplicity),

$$
f_{t}^{4} \frac{1}{m_{t}^{2}}=\left(g \frac{m_{t}}{M_{W}}\right)^{4} \frac{1}{m_{t}^{2}}=g^{4} \frac{m_{t}^{2}}{M_{W}^{4}}
$$


In fact, our calculation showed it is really the case. Namely the coefficient function of the 4-Fermi operator to describe the box diagram is known to behave as

$$
E\left(x_{t}\right) \simeq-\frac{1}{4} x_{t} \quad\left(\text { for } x_{t} \gg 1 \quad\left(x_{t} \equiv \frac{m_{t}^{2}}{M_{W}^{2}}\right)\right)
$$

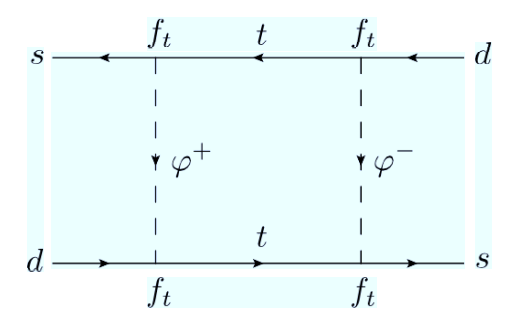

Figure 4: The top quark contribution to the box diagram

One comment here is that there is a qualitative difference between FCNC Z and $\gamma$ vertices. In the case of $Z$ vertex (see Fig.5), just as in the box diagram, we expect to have non-decoupling effect proportional to $m_{t}^{2}$ :

$$
g f_{t}^{2}=g^{3} \frac{m_{t}^{2}}{M_{W}^{2}},
$$

which is confirmed by our explicit calculation, whose result was given in the form of vertex function,

$$
C\left(x_{t}\right) \simeq \frac{1}{4} x_{t} \quad\left(\text { for } x_{t} \gg 1\right) .
$$

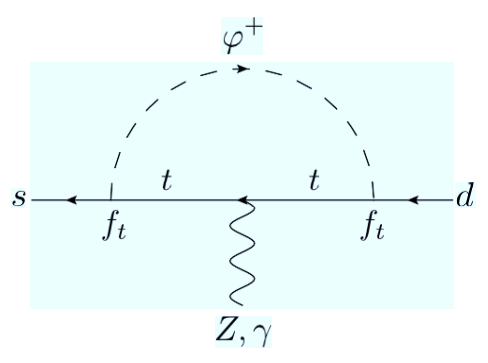

Figure 5: Flavor changing $\gamma$ and $\mathrm{Z}$ vertices

On the other hand, in the case of $\gamma$ vertex (see Fig.5), the CVC (conserved vector current) necessitates an extra factor $q^{2} \gamma_{\mu}-q_{\mu} q$ and the remaining vertex function is expected to behave as

$$
e f_{t}^{2} \frac{1}{m_{t}^{2}} \log m_{t}=\frac{e g^{2}}{M_{W}^{2}} \log m_{t}
$$

which was again confirmed by our calculation: the vertex function $F_{1}$ shows such logarithmic behavior. Intuitively speaking, to some extent the FCNC $\gamma$ vertex mimics QED, where decoupling theorem exactly holds. 
Thus the contributions of 4th generation, if it ever exists, may be remarkable in the $\mathrm{Z}$ vertex (weak-penguin diagram) rather than in the $\gamma$ vertex.

\section{CP violation in the Kobayashi-Maskawa model and the non-decoupling effects}

It was great news that the Nobel prize in physics of 2008 was awarded to Professors Nambu, Kobayashi and Maskawa.

In the Kobayashi-Maskawa (KM) model, $\mathrm{CP}$ violation is accompanied by the breaking of flavor symmetry. Namely, if there is mass degeneracy between different generations, CP violation disappears even for non-vanishing $\mathrm{CP}$ violating phase. Intuitively, in this case the number of generations is effectively reduced. Hence, $\mathrm{CP}$ violating observables are induced through FCNC processes.

This argument suggests that to get $\mathrm{CP}$ violation in the $\mathrm{KM}$ model, all of three generations have to participate. In fact, in the neutral kaon system made of first two generations, CP violating observable $\varepsilon_{k}$ is dominated by the non-decoupling effect due to t quark:

$$
\varepsilon_{k} \propto \operatorname{Im}\left\{\left(V_{t s}^{*} V_{t d}\right)^{2}\right\} E\left(x_{t}\right) \propto \operatorname{Im}\left\{\left(V_{t s}^{*} V_{t d}\right)^{2}\right\} \frac{m_{t}^{2}}{M_{W}^{2}} .
$$

As far as all three generations participate, $\mathrm{CP}$ violating observables should appear not only kaon system but also in the $\mathrm{B}$ meson system. In addition, since the non-decoupling effect due to the top quark is the dominant source, such CP violating effect is expected to be large in the B system, which couples to the t quark more directly, as was pointed out by A.I. Sanda et al. [8] This kind of reasoning led to the B-factory experiments.

\section{The ideas of new physics and the non-decoupling effects}

So far we have been discussing the flavor physics mainly in 1980's. Finally, let us turn to recent topics in flavor physics. Namely, there seem to be a few "puzzles" suggested by recent experimental data, which I would like to discuss very briefly below.

(1) Rather large rate of $D^{0} \leftrightarrow \bar{D}^{0}$ mixing

Recently rather large mixing rate of $D^{0} \leftrightarrow \bar{D}^{0}$ has been observed,

$$
x_{D} \equiv \frac{\Delta M_{D}}{\Gamma_{D}}=(0.811 \pm 0.334) \cdot 10^{-2}(\mathrm{HFAG}),
$$

while the short distance contribution of the Standard Model is estimated to be very small due to small mass-squared differences among $\mathrm{d}$, s and b quarks:

$$
x_{D}(S D) \sim 10^{-6}-10^{-4} .
$$

(2) Possible deviation from the Standard Model predictions for CP asymmetries in B decays (see, e.g., (9):

(i) The CP asymmetry observable in B factories, $\sin 2 \beta\left(\sin 2 \phi_{1}\right)$, predicted by use of $\varepsilon_{k}$ and $\frac{\Delta M_{s}}{\Delta M_{d}}$ is a little larger than directly measured value by $B \rightarrow \psi K_{S}$. 
(ii) There seems to exist rather large difference in the direct $\mathrm{CP}$ asymmetries, which are naively expected to be identical from isospin analysis:

$$
\Delta A_{C P}=A_{C P}\left(B^{-} \rightarrow K^{-} \pi^{0}\right)-A_{C P}\left(B^{0} \rightarrow K^{-} \pi^{+}\right)=(14.4 \pm 2.9) \% .
$$

(iii) In addition, there is an indication of large non-standard CP phase in the $B_{s}$ decay, $B_{s} \rightarrow \psi \phi$.

Among various possibilities of NP, the presence of fourth family may be a natural cause of these puzzles. The contributions of possible 4th family to FCNC and $\mathrm{CP}$ violating processes were discussed in the pioneering work long time ago [10]. Since the heavy fermions of fourth generation should get their masses through SSB, they yield non-decoupling effects enhanced as $m_{t^{\prime}}^{2} / m_{t}^{2}$, compared with those by third generation, except for the factors of generation mixings. The enhancements by the powers of $m_{t^{\prime}}^{2}$ will be realized in the box diagram and FCNC Z vertex (weak penguin), thus making the characteristic predictions of the scenario possible.

\section{Summary}

We summarize the issues covered in this talk below.

(1) The question of whether heavy fermions decouple from low energy processes or not was an interesting theoretical issue in early 80 's.

(2) The top quark turned out to be unexpectedly heavy, and was found to have important nondecoupling contributions to FCNC processes, including CP violating processes, as the reflection of the spontaneous gauge symmetry breaking.

(3) The extent of the non-decoupling effect is different in QED- and weak-penguin diagrams.

(4) In the Kobayashi-Maskawa model, CP violating observables are inevitably induced by FCNC processes.

(5) The non-decoupling effects of the top quark play crucial roles in the $\mathrm{CP}$ violating observables both in $\mathrm{K}$ and $\mathrm{B}$ meson systems.

(6) Recent claims on the possible deviations from the Standard Model predictions concerning $D^{0} \leftrightarrow$ $\overline{D^{0}}$ and CP asymmetries in B decay processes may signal some new physics (NP).

(7) If we take the possibility of the 4th generation as a candidate of NP, the unknown heavy fermions of 4th generation are expected to have enhancement factor, proportional to $m_{t^{\prime}}^{2}$.

(8) The fact that the enhancement is remarkable in the box and weak-penguin diagrams may make characteristic predictions of the 4th generation scenario possible.

\section{Acknowledgment}

I would like to express my sincere gratitude to Professor Takeo Inami for the fruitful and enjoyable collaboration, which my talk was based upon.

\section{References}

[1] T. Inami and C. S. Lim, Prog. Theor. Phys. 65, 297 (1981).

[2] S.L. Glashow, J. Iliopoulos and L. Maiani, Phys. Rev. D2, 1285 (1970). 
[3] M.K. Gaillard and B.W. Lee, Phys. Rev. D10, 897 (1974).

[4] M. Kobayashi and T. Maskawa, Prog. Theor. Phys. 49, 652 (1973).

[5] K. Fujikawa, B.W. Lee and A.I. Sanda, Phys. Rev. D6, 2923 (1972).

[6] A.J. Buras, Phys. Rev. Lett. 46, 1354 (1981).

[7] T. Appelquist and J. Carrazone, Phys. Rev. D11, 2856 (1975).

[8] I.I. Bigi and A.I. Sanda, “CP Violation", Cambridge Univ. Press (2000).

[9] A. Soni, A.K. Alok, A. Giri, R. Mohanta, S. Nandi, arXiv: 0807.1971[hep-ph].

[10] W.-S. Hou, R.S. Willey and A. Soni, Phys. Rev. Lett. 58, 1608 (1987); W.-S. Hou, A. Soni and H. Steger, Phys. Rev. Lett. 59, 1521 (1987); W.-S. Hou, A. Soni and H. Steger, Phys. Lett. B192, 441 (1987). 